XXI Congreso de Ingeniería de Organización

Valencia, Spain, July 5-6, 2017. The final publication is available at Springer via

http://dx.doi.org/10.1007/978-3-319-96005-0 41

\title{
A Simulink library for supply chain simulation
}

\author{
Trapero JR ${ }^{1}$, Ramos F², Holgado de Frutos E
}

\begin{abstract}
Despite the links between forecasting and inventory control, both areas have evolved separately. This work presents a supply chain simulation library developed in Simulink to bridge that gap. To simulate a supply chain is important to define the number of companies/echelons that belong to the same supply chain and the policies that each company employs to demand forecasting and stock control. The potential user can find in this library forecasting and stock control blocks to simulate a supply chain in a modular design. We show how to implement: i) Forecasting policies. For example, the widely used exponential smoothing; ii) Replenishment models. For instance, typical order-up-to-level stock control policies as $(s, S)$; and finally, how to connect the forecasting and stock control blocks to describe the performance of a company and to extend such relationships to define the whole supply chain.
\end{abstract}

Keywords: Supply chain simulation; forecasting; inventory control.

\section{Introduction}

Traditional operations management textbooks (Nahmias and Olsen., 2015) widely used in supply chain courses analyse separately the areas of forecasting and stock

\footnotetext{
${ }^{1}$ Juan Ramón Trapero ( e-mail: juanramon.trapero@uclm.es)

Dept. of Business Administration. Facultad de Ciencias y Tecnologías Químicas. Universidad de Castilla-La Mancha. Avda. Camilo José Cela S/N, 13071Ciudad Real, Spain

${ }^{2}$ Francisco Ramos (e-mail: Francisco.Ramos@uclm.es)

Dept. of Electrical, Electronics, Automatica and Communications Engineering. E.T.S. de Ingenieros Industriales. Universidad de Castilla-La Mancha. Avda. Camilo José Cela S/N, 13071Ciudad Real, Spain

${ }^{3}$ Enrique Holgado de Frutos (e-mail: enrique.holgado.de.frutos@gmail.com)

Dept. of Business Administration. Facultad de Ciencias y Tecnologías Químicas. Universidad de Castilla-La Mancha. Avda. Camilo José Cela S/N, 13071Ciudad Real, Spain
} 
control. Usually, different forecasting techniques are explained to provide optimal forecasts depending on the demand features as trend and/or seasonality. For instance, the family of exponential smoothing methods, ARIMA models, etc, (Ord and Fildes, 2013). On the other hand, stock control techniques are defined assuming different hypothesis about the demand as stationarity, independence, and known statistical distribution, which typically is chosen Gaussian (Nahmias and Olsen., 2015). Although, it is acknowledged that forecasting errors are a key variable to determine the safety stocks and the reorder points in well-known stock control policies like order-up-to-level, from the authors' point of view it is not clear how these two areas are connected and it may be a reason behind their isolation from each other.

To bridge that gap this work presents a simulation library developed in SIMULINK that intends to facilitate the task of simulating supply chains to improve decision making. In this sense, this library presents a system dynamics structure with a modular/block design. In other words, the main policies as the forecasting technique and the inventory control method are expressed as a set of inputs/outputs logically connected. Furthermore, in case the user requires trying either different forecasting policies or inventory policies, he/she just ought to change the corresponding block regardless of the rest of the supply chain model.

This supply chain simulation library is useful for both academics and practitioners. Academics can use this library to introduce students to topics as demand forecasting and stock control and, more importantly, to remark the links between both areas. Additionally, practitioners can be benefited from this library because it can be easily utilized to simulate their supply chains and, for example, to analyse what if possibilities to improve their decision making.

The article is structured as follows. Section 2 describes the process to implement the forecasting techniques in Simulink/Matlab, particularly, the exponential smoothing forecasting method. Section 3 explains the implementation of an orderup-to-level stock control policy. Section 4 determines the inputs/outputs of the forecasting and stock control blocks and how they are linked in a system dynamics fashion. Section 5 carries out several simulations to verify the correct performance of the supply chain model, and finally, last section summarizes the main conclusions of the work.

\section{Demand forecasting}

Demand forecasting is a key part of supply chain modeling, particularly for those companies where such forecasts drive the manufacturing planning and control. For the sake of simplicity, here we will implement a simple exponential smoothing (SES) technique given its widely use (Ord and Fildes, 2013), although more complex forecasting techniques can also be implemented.

The forecasts provided by SES are computed as follows: 


$$
F_{t+1}=\alpha Y_{t}+(1-\alpha) F_{t}
$$

Since observations are available up to time $t, F_{t+1}$ is the one step ahead forecast, $Y_{t}$ is the actual demand observation at time $t$, and $\alpha$ is the exponential smoothing constant that varies between 0 and 1 . In case that the forecasting step is smaller than the lead time, the lead time demand forecast is defined as:

$$
F_{L}=\sum_{i=1}^{L} F_{t+i}=L \cdot F_{t+1}
$$

Often, the forecasting step is weekly and the lead time comprises several weeks. Moreover, when using SES it can be shown that $F_{t+i}=F_{t+1}$ for $i=1,2, \ldots, L$. (Ord and Fildes, 2013). It should be noted that, the lead time $(L)$ is assumed to be known and constant. Fig. 1 depicts a possible SES implementation, where alphal is $\alpha$ in equation (2.1) and $\mathrm{Z}^{-1}$ is the delay operator (Z-transform) such as $F_{t+1} \cdot Z^{-1}=F_{t}$

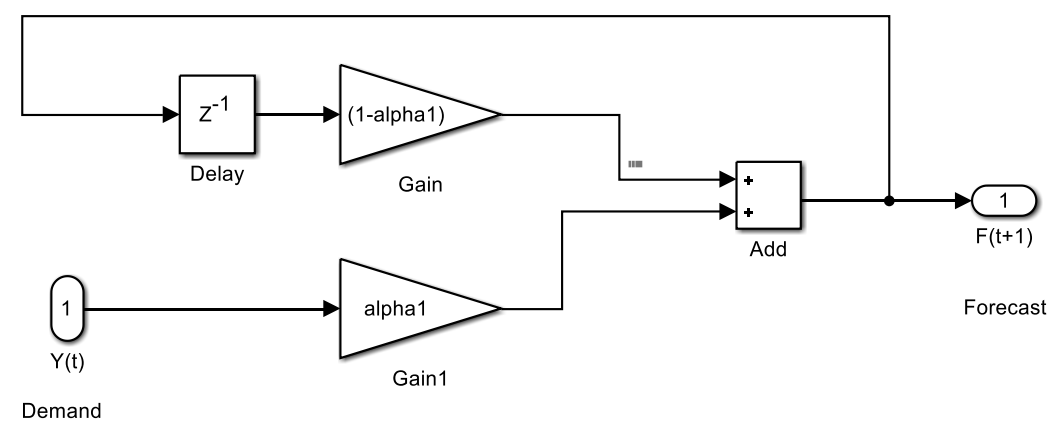

Fig. 1 Simple Exponential Smoothing implemented in Simulink.

Equation (2.1)-(2.2) shows the point lead time demand forecast, i.e., the mean estimate of the lead time demand forecast. However, some stock control policies may also require a measure of the forecasting error uncertainty from the forecasting system to compute the safety stock. According to (Silver et al., 1998) if the forecasting errors are Gaussian independent and identically distributed (iid), the safety stock $(S S)$ for a target cycle service level $(C S L)$ can be expressed as:

$$
S S=k \sigma_{L}
$$

where $k=\Phi^{-1}(1-C S L)$ is the safety factor; $\Phi(\cdot)$ is the standard normal cumulative distribution function and $\sigma_{L}$ is the lead time forecast of the forecasting error 
standard deviation. Here, the main problem is how to compute $\sigma_{L}$ from the forecasting error. A common approach is firstly, to compute $\sigma_{1}$ (one step ahead forecast of the forecasting error standard deviation) based on the Mean Squared Errors (MSE) and then, to estimate $\sigma_{L}$ (Silver et al., 1998). In this sense, MSE at time $t$ is:

$$
M S E_{t}=\frac{1}{n} \sum_{t=1}^{n}\left(Y_{t}-F_{t}\right)^{2}
$$

Since the errors variability measured by $M S E_{t}$ may not be constant, it is possible to apply a second SES to $M S E_{t}$ such as:

$$
M S E_{t+1}=\alpha^{\prime} Y_{t}+\left(1-\alpha^{\prime}\right) M S E_{t}
$$

and estimate $\sigma_{1}=\sqrt{M S E_{t+1}}$. Secondly, an exact relationship between $\sigma_{1}$ y $\sigma_{L}$ was found in (Graves, 1999), such as:

$$
\sigma_{L}=\sigma_{1} \sqrt{L} \sqrt{1+\alpha(L-1)+\frac{1}{6} \alpha^{2}(L-1)(2 L-1)}
$$

Fig. 2 shows the forecasting system that provides the lead time demand quantile forecast: $F_{L}+k \sigma_{L}$, where the last term $\left(k \sigma_{L}\right)$ is the safety stock.

\section{Stock control policy}

Once the forecasting policy is defined, the resulting forecasts are fed to the stock control system. Here, we will implement a continuous review, reorder point, order-up-to-level $(s, S)$ policy (Silver et al., 1998), although the implementation of other stock policies like $(S-1, S),(s, Q)$ is straightforward. 


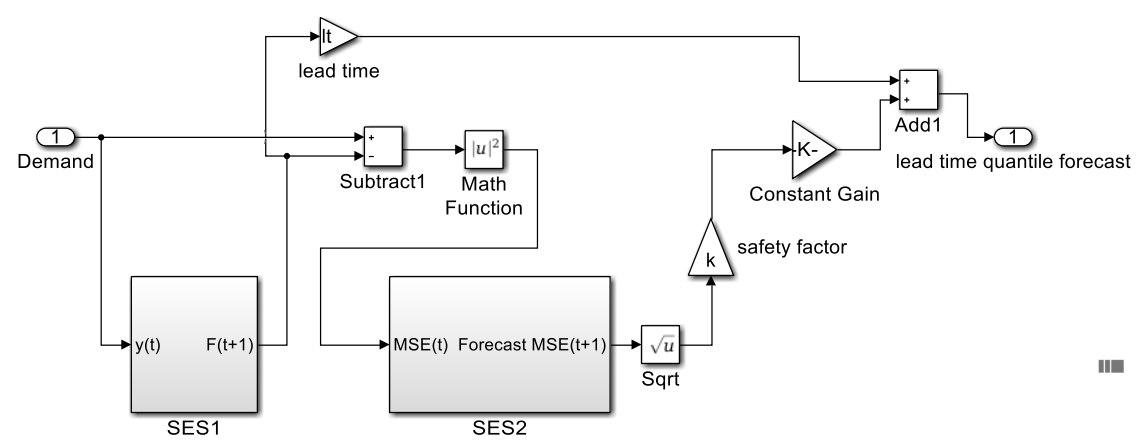

Fig. 2 Forecasting system with two SES. SES1 for the lead time mean forecast and SES2 for the safety stock.

Let's assume that we are interested in modelling a retailer with a SES as a forecasting technique and a $(s, S)$ stock control policy. Fig. 3 shows that retailer implemented in Simulink.

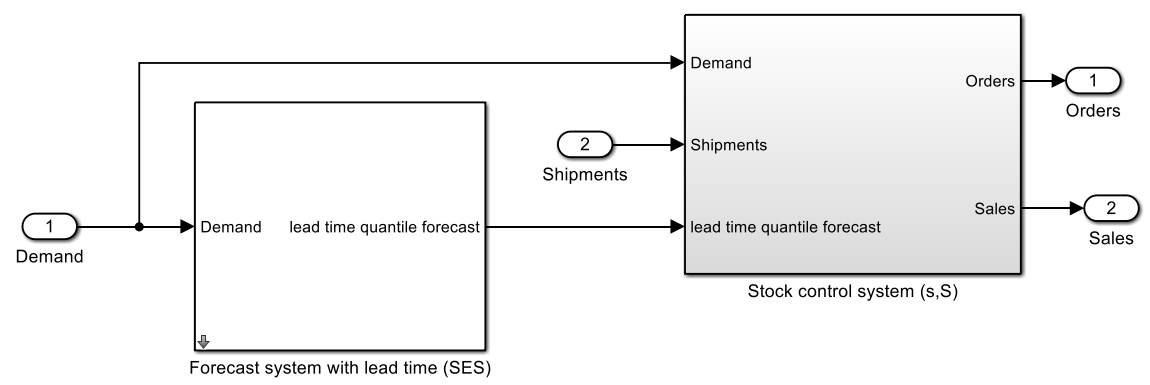

Fig. 3 Retailer that uses SES and $(s, S)$ as forecasting technique and stock control, respectively.

We have followed a similar block design developed by (Dejonckheere et al., 2003). Focusing on the stock control system, its inputs are: i) Demand; ii) Shipments, i.e., receptions received from an upwards echelon as a manufacturer; iii) Lead time quantile forecast. This signal is obtained from the forecasting system showed in Fig. 2. Recall that such a lead time demand quantile forecast is the reorder point $(s)$, i.e.:

$$
s=F_{L}+k \sigma_{L}
$$

The outputs of the stock control system are: i) Orders; and ii) Sales. Fig. 4 depicts how those outputs are calculated. In summary, the retailer satisfies the market demand and with the shipments received updates the Net Stock (NS). Any unfilled demand is backlogged in our model. The Work In Process (WIP) is also updated with the orders and shipments. The Inventory Position (IP) is computed as the sum 
of NS and WIP. Finally, the IP is compared with the reorder point $(s)$ and if IP is lower than $s$, then the retailer launches an order, such as:

$$
O_{t}=S-I P_{t}
$$

where $S$ is the order-up-to-level.

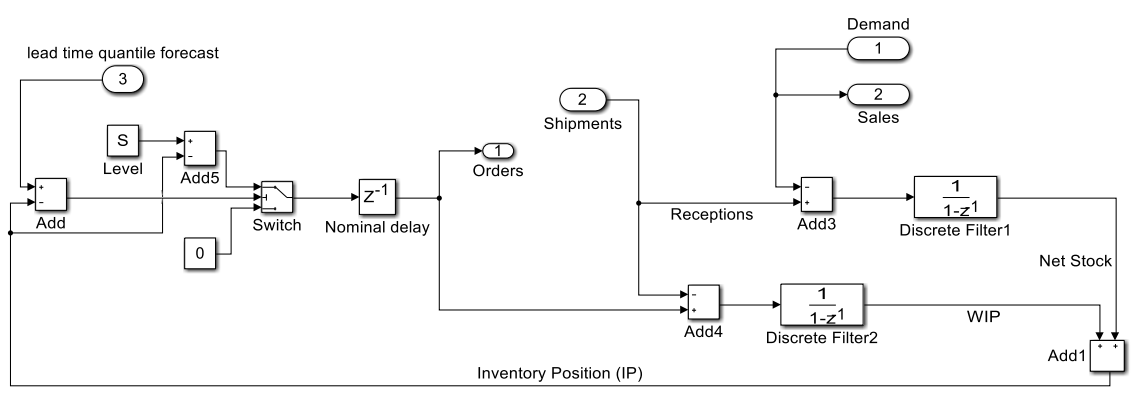

Fig. 4 Block diagram of the stock control system $(s, S)$.

\section{Supply chain dynamics}

In the previous sections, we have explained the links between the forecasting system and the stock control. In this section, we will use such blocks to simulate a supply chain comprised of a retailer and a manufacturer. Fig. 5 shows the retailer system described in Figs. 3 and 4, where only the set of inputs/outputs are visible.

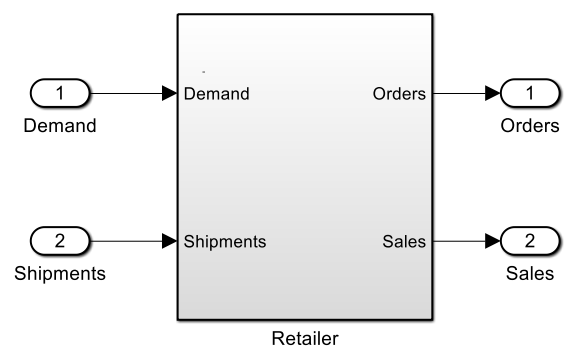

Fig. 5 Example of a retailer system where only inputs and outputs are visible.

To simulate the supply chain, we should connect those inputs/outputs with the rest of echelons. Fig. 6 shows the supply chain. The first block is the market that generates the demand and receives sales from retailer. Fig. 7 shows the market, where the demand is built as a random number generator, although it can be easily changed for a file with actual demand data. Fig. 6 also shows the retailer and 
manufacturer subsystems. The retailer has been previously described in Figs. 3-5. The manufacturer follows the same structure than the retailer with two slight differences. Since the manufacturer does not order to upstream supply chain members, that subsystem does not have an orders output. Nonetheless, it requires some time to produce the goods ordered by the retailer, which is modeled by the manufacturing time, see Fig. 8.

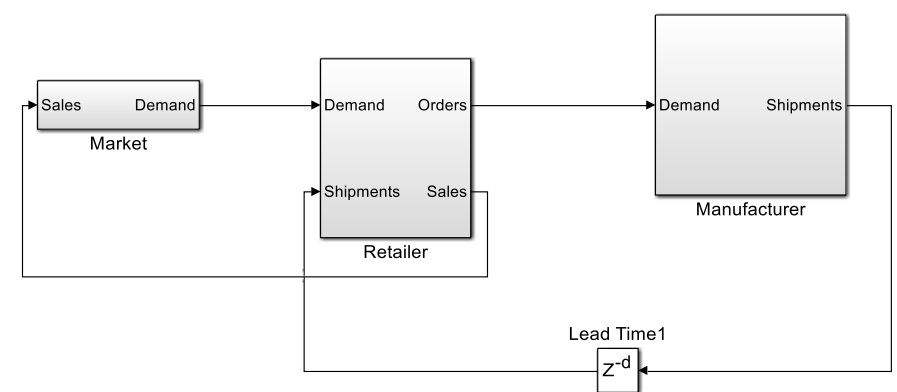

Fig. 6 Example of supply chain with two members serially connected.
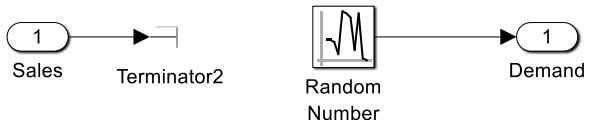

Fig. 7 Market subsystem.

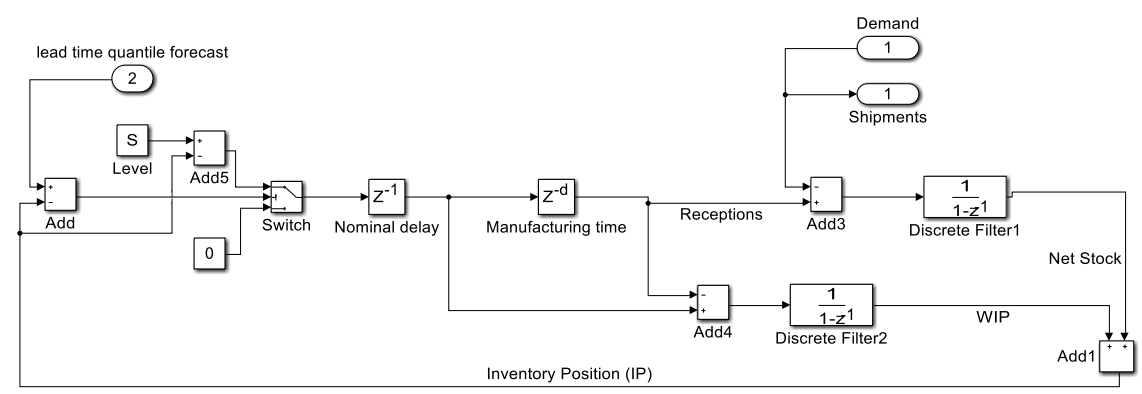

Fig. 8 Block diagram of the stock control system $(s, S)$ for the manufacturer.

\section{Simulation results}

To verify the correct model performance, some simulations were carried out. The market demand was simulated with a normal random number generator with mean 100 and variance 20 . The retailer parameters were set to $\alpha=0.1, \alpha^{\prime}=0.01$, $C S L=95 \%, L=3, S=1000$. The manufacturer parameters were set to $\alpha=0.1$, 
$\alpha^{\prime}=0.01, C S L=95 \%, L=3, S=2000$. Fig. 9 shows some results for the retailer. Left panel depicts the forecasting results for the mean and variance forecasts. Right panel shows stock control results. The figure shows the forecasts (left plots) and inventory variables (right plot).
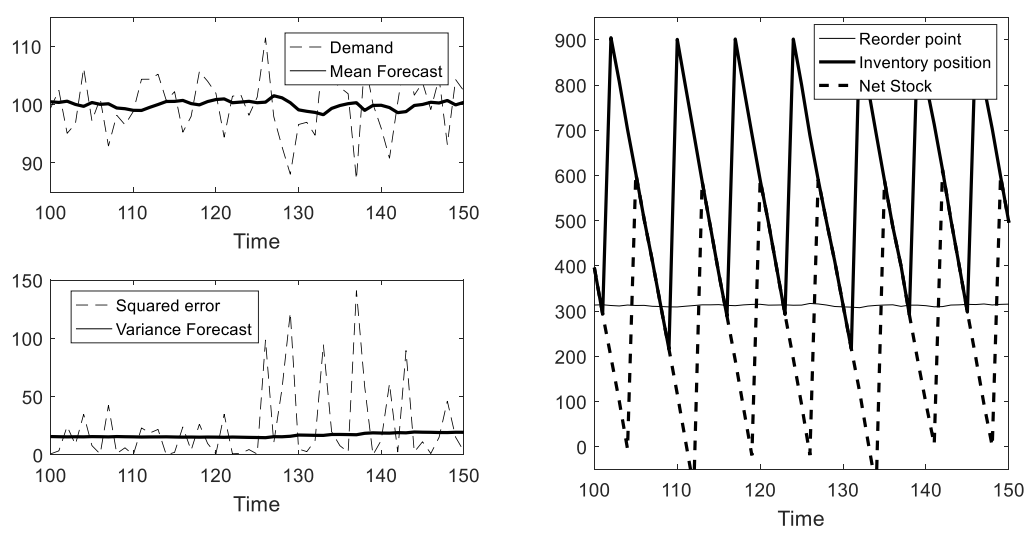

Fig. 9 Simulation results for the retailer.

\section{Conclusions}

This work aims at developing a simulation library to facilitate the understanding of supply chains. Following the described methodology, different forecasting techniques and stock control policies can be easily implemented. This library can be useful for teaching supply chain concepts and, particularly, improves the links between forecasting and inventory control. Additionally, practitioners can implement their supply chains to improve their decision-making process.

\section{References}

Dejonckheere J, Disney SM, Lambrecht MR, Towill DR (2003) Measuring and avoiding the bullwhip effect: A control theoretic approach. European Journal of Operational Research $147: 567-590$

Graves SC (1999) A single-item inventory model for a nonstationary demand process. Manufacturing \& service operations management 1:50-61

Nahmias S, Olsen TL (2015) Production and operations analysis. Waveland Press.

Ord K, Fildes R (2013) Principles of business forecasting. Cengage Learning.

Silver E, Pyke D, Peterson R (1998) Inventory management and production planning and scheduling. Wiley. 